Revue d'études américaines. American Studies Journal

$1 \mid 2015$

The Voting Rights Act at 50 / Hidden in Plain Sight:

Deep Time and American Literature

\title{
Cécile Cottenet (ed.), Race, Ethnicity and Publishing in
} America

\section{Sandrine Ferré-Rode}

\section{CpenEdition}

\section{Journals}

Electronic version

URL: https://journals.openedition.org/transatlantica/7288

DOI: $10.4000 /$ transatlantica.7288

ISSN: $1765-2766$

\section{Publisher}

Association française d'Etudes Américaines (AFEA)

\section{Electronic reference}

Sandrine Ferré-Rode, "Cécile Cottenet (ed.), Race, Ethnicity and Publishing in America", Transatlantica

[Online], 1 | 2015, Online since 20 December 2019, connection on 08 February 2023. URL: http://

journals.openedition.org/transatlantica/7288 ; DOI: https://doi.org/10.4000/transatlantica.7288

This text was automatically generated on 8 February 2023.

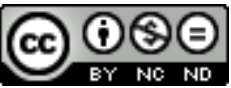

Creative Commons - Attribution-NonCommercial-NoDerivatives 4.0 International - CC BY-NC-ND 4.0

https://creativecommons.org/licenses/by-nc-nd/4.0/ 


\title{
Cécile Cottenet (ed.), Race, Ethnicity and Publishing in America
}

\author{
Sandrine Ferré-Rode
}

\section{REFERENCES}

COTTENET, Cécile (ed), Race, Ethnicity and Publishing in America, London, New York, Palgrave-Macmillan, 2014, 264 p., ISBN : 978-1-137-39051-6, £ 55

1 Cet ouvrage, publié sous la direction de Cécile Cottenet, constitue le prolongement d'un colloque international organisé à l'université Aix-Marseille en 2012. Il vient enrichir un domaine de recherche multidisciplinaire pour lequel chercheurs français et étrangers montrent depuis plusieurs années un intérêt grandissant. En effet, les études présentées se situent à la croisée des disciplines de l'histoire du livre et des études ethniques (notamment les études afro-américaines) ${ }^{1}$, mais aussi des gender studies et de la littérature. L'ouvrage se compose de dix articles en anglais regroupés en quatre parties, elles-mêmes encadrées par une introduction critique de Cécile Cottenet et un épilogue de Samuel Blumenfeld. L'introduction permet d'aborder l'ouvrage en ayant au préalable accès à une historiographie de la discipline dans laquelle il vient s'insérer et à une présentation des articles qui permet de percevoir les liens thématiques et méthodologiques qui les unissent. Tous les auteurs ont aussi visiblement veillé, par le biais de multiples comparaisons et renvois internes, à inscrire leur contribution au sein d'un ensemble qui s'articule de manière cohérente, dans une optique de complémentarité, de nuance et de progression du propos.

2 Dans la première partie intitulée "Historiography ", les articles de Claire Parfait et de Cheryl Knott montrent à quel point les préjugés raciaux aux États-Unis ont pu limiter l'impact de publications historiques. Ainsi, l'article que Claire Parfait consacre à l'historien noir William Cooper Nell (1816-1874) analyse l'histoire éditoriale complète (depuis la publication, la promotion, la diffusion jusqu'à la réception) de l'œuvre majeure de Nell consacrée aux Patriotes noirs tombés pendant la Révolution 
américaine. D'abord contenus dans de brefs pamphlets publiés en 1851 et 1852 sous le titre Services of Colored Americans in the Wars of 1776 and 1812, les travaux historiques de Nell - surtout de nature militaire et biographique - devinrent un ouvrage complet de quelque 400 pages intitulé Colored Patriots of the American Revolution et publié en 1855. Clairement, l'objectif de Nell était de fournir aux Américains, noirs et blancs, un «passé utile » (« a usable past » 32) en rectifiant l'omission du rôle des Noirs dans la Révolution américaine et en permettant aux Afro-Américains de se forger une identité fondée sur une fierté collective. Si Claire Parfait montre bien que la visée politique et didactique de Nell était commune à d'autres historiens noirs de l'époque, l'examen de son œuvre en tant qu'objet matériel permet de mieux appréhender sa portée politique, identitaire et culturelle, mais aussi son importance historiographique. Nell dut publier ses œuvres à compte d'auteur et en assurer seul la promotion. De même, bien que Colored Patriots fit l'objet de nombreuses recensions dans la presse du Nord et du Sud, l'historien ne parvint pas à en tirer un quelconque profit financier, notamment en raison du manque de soutien des Afro-Américains eux-mêmes, encore trop timidement enclins à la lecture, soit par illettrisme, soit par incapacité économique à acheter des livres. De ce fait, Nell et son œuvre ont rapidement disparu de l'histoire dominante, écrite et prescrite par la société blanche. L'article de Cheryl Knott, quant à lui, permet au lecteur une incursion dans le domaine très spécialisé de l'histoire des bibliothèques, notamment dans la première moitié $\mathrm{du} \mathrm{xx}^{\mathrm{e}}$ siècle. Durant cette période de l'histoire des États-Unis, les établissements publics se multiplient, notamment grâce aux fonds de la fondation Carnegie, mais leur accès au public afro-américain demeure interdit ou restreint en raison de la politique ségrégationniste en vigueur dans de nombreux États du Sud. En reconstituant l'histoire de la publication, de la diffusion et surtout de la réception de l'ouvrage intitulé The Southern Negro and the Public Library, publié en 1940 par Eliza Atkins Gleason, Cheryl Knott démontre comment et pourquoi les bibliothèques américaines, bien qu'institutions publiques, ont échoué dans leur mission démocratique de faciliter l'accès à la connaissance de l'ensemble des citoyens américains. L'article retrace le parcours personnel et professionnel d'Eliza Atkins, première femme noire américaine à obtenir un doctorat en bibliothéconomie avec une thèse soutenue à l'université de Chicago en 1940. Ce sont les travaux menés pour cette thèse que reprend l'ouvrage The Southern Negro and the Public Library, lequel propose une topographie des bibliothèques, une étude des différents types de services de bibliothèques accessibles ou non aux Noirs, mais aussi des recommandations sur l'amélioration possible de ces services. Cheryl Knott démontre comment l'ouvrage, quoique particulièrement novateur, et en dépit de multiples recensions dans la presse spécialisée, ne rencontra pourtant que peu d'écho dans le Sud des États-Unis, qui resta sourd aux suggestions subtilement faites par l'auteur de mettre un terme à la ségrégation dans ces lieux d'apprentissage et de partage des savoirs que sont les bibliothèques.

3 Les articles qui composent la deuxième partie intitulée «Bilingualism and Ethnic Identity" s'intéressent à la manière dont les écrivains d'une minorité ethnolinguistique peuvent parvenir à construire une visibilité pour leurs œuvres et pour euxmêmes au sein d'un pays dont la production littéraire et culturelle les maintient à la marge. Manuel Brito décrit le rôle primordial de plusieurs maisons d'édition hispaniques, notamment Bilingual Review Press et UCLA Chicano Studies Research Center Press, dont la politique éditoriale repose sur la publication d'œuvres littéraires et nonlittéraires exclusivement hispaniques. Ces éditeurs pérennisent leur influence grâce à 
une forme d'institutionnalisation centrée sur la publication de revues littéraires phares, et sur la création et le soutien de programmes universitaires d'études hispaniques aux États-Unis. Une partie substantielle de l'exposé est consacrée à l'émergence de ces maisons d'édition dans le contexte du mouvement des droits civiques et du Chicano movement des années 1960 et 1970. On regrettera que cette approche historique, quoique nécessaire, prenne parfois le pas sur une analyse esthétique et thématique plus poussée de la production littéraire contemporaine des années 2000 (le titre de l'article évoque pourtant "new Hispanic texts»), analyse qui aurait peut-être permis de percevoir en quoi cette production diffère, ou non, de celle des années «fondatrices ». Manuel Brito montre bien comment ces maisons d'édition ont joué et continuent de jouer un rôle éminemment politique et social, en fournissant aux écrivains non seulement une vitrine mais aussi un certain nombre de leviers sociaux, culturels et économiques dont toute la communauté hispanique des États-Unis bénéficie. Néanmoins, on pourra regretter que la question du bilinguisme des auteurs et surtout de leurs œuvres ne soit plus longuement traitée, par exemple en privilégiant une brève étude de contenu ou l'analyse du parcours de quelques auteurs représentatifs, afin de mieux distinguer l'expérience hispanique de celle d'autres groupes ethniques présents aux États-Unis. C'est cette double approche que privilégie Peggy Pacini dans l'article qu'elle consacre aux auteurs dits franco-américains, essentiellement des Canadiens-Français ayant immigré en Nouvelle-Angleterre depuis le Québec au début du xx siècle, et parmi lesquels on citera Rhea Côté Robbins et David Plante. En mettant la question de la langue d'écriture, anglais ou français, au cœur de sa réflexion, Peggy Pacini expose clairement l'ambiguïté de la quête de visibilité des auteurs franco-américains: le choix d'écrire dans la langue dominante, l'anglais, représente un risque réel de perte d'identité, d'assimilation et, finalement, d'invisibilité ; le choix de la langue française, en revanche, (op)pose l'identité " autre " en rempart, réduisant drastiquement l'accès potentiel aux marchés littéraires nationaux et internationaux. Finalement, l'auteur étiqueté "franco-américain », qui peut certes bénéficier d'une « infrastructure » lui permettant d'accéder à la publication grâce à l'existence et à la vitalité de maisons d'édition régionales, d'associations d'écrivains, de programmes d'études universitaires et de revues littéraires spécialisées, se retrouve dans un espace créatif interstitiel : «in-visible, visible from the inside, visible in-between » (112). De ce fait, l'œuvre franco-américaine ne peut accéder ni au canon national américain, ni au canon francophone essentiellement québécois (situation qui, incidemment, n'est pas sans rappeler la situation de la littérature acadienne au Canada).

Dans la troisième partie de l'ouvrage intitulée «Challenging Stereotypes: A Gendered Perspective ", le choix de juxtaposer l'article de Claudine Raynaud, qui offre une lecture critique de l'essai de Zora Neale Hurston "What White Publishers Won't Print » publié en 1950 dans la revue Negro Digest, et celui de Matilde Martín González qui s'intéresse à l'impact de la création de maisons d'édition publiant des écrivains "féministes du Tiers-Monde » est singulièrement réussi. A priori traitant de deux thématiques différentes, les deux articles se font écho dans leur analyse de la manière dont un objectif commun, celui de combattre les stéréotypes et de donner une voix audible à un groupe marginalisé, peut être atteint par des stratégies différentes. Claudine Raynaud montre ainsi comment et avec quelles difficultés les choix esthétiques de Zora Neale Hurston marquent son refus de faire de la question raciale, surtout dans sa dimension sociologique, la raison d'être de son œuvre littéraire. D'une part, Hurston critiquait la 
vision stéréotypée des Noirs véhiculée notamment, mais pas exclusivement, par des écrivains (et leurs éditeurs) blancs, rejetant ces modèles établis qui faisaient du héros noir soit un être exceptionnel, soit un personnage caricatural. Si elle revendiquait la liberté, voire la nécessité, de faire de l'homme noir "ordinaire », issu de la classe moyenne, un héros fictionnel, Hurston aspirait également à produire une littérature plus humainement universelle. Cette double forme d'émancipation d'une tradition littéraire réductrice permettait par ailleurs à Hurston d'envisager que son œuvre fictionnelle puisse intégrer le mouvement moderniste de l'époque, et de ce fait atteindre un public plus large. Ainsi les succès, mais aussi les querelles, les compromis et les revers de l'écrivaine noire aux prises avec le petit monde de l'édition dominé par les Blancs sont analysés avec finesse au prisme des idéaux défendus par Hurston, par ailleurs souvent mal perçus dans le contexte très conservateur des années 1950. Replacé dans une chronologie détaillée de la carrière de l'auteur, comparé à d'autres essais de Hurston sur des thématiques similaires et confronté aux positions parfois ambiguës de son auteur, "What White Publishers Won't Print » révèle tout son sens, sa subtilité et sa portée. C'est une tout autre stratégie d'accès à la visibilité que décrit Matilde Martín González dans son article sur les petites maisons d'édition créées dans les années 1980 pour publier les œuvres de femmes issues de minorités ethniques, sociales ou sexuelles. Une partie de l'analyse expose le contexte dans lequel sont apparues, entre autres, des maisons comme Kitchen Table: Women of Color Press, Third Woman Press et Aunt Lute Press. Ainsi, face au recul des acquis politiques, économiques et sociaux des femmes et des féministes constaté dans les années 1980, ces maisons d'édition ont pu ouvrir l'accès à la publication à des auteurs marginalisées, par le biais d'une politique éditoriale élaborée sans tenir compte des paramètres de rentabilité régissant les entreprises commerciales. Par ailleurs, elles ont permis de créer un réseau crucial en réunissant des femmes qui ont toutes en commun l'expérience de la minorisation raciale. Enfin, en s'attardant sur quelques auteurs, anthologies et œuvres phares, l'article montre comment cette littérature minoritaire peut enrichir et élargir le canon littéraire américain, dont il est précisément question dans la dernière partie de l'ouvrage.

5 «Re-visiting the Canon » regroupe trois contributions qui permettent un retour sur des œuvres canoniques de la littérature américaine en s'intéressant non pas tant à la difficulté de leurs auteurs à parvenir à la publication, mais en mettant en lumière d'autres aspects du long parcours du texte au livre. Ainsi John K. Young choisit-il de s'arrêter sur ce que l'on peut considérer comme une phase préliminaire de l'existence de l'œuvre majeure de Jean Toomer, Cane, publiée en 1923 chez l'éditeur new-yorkais Boni \& Liveright. En effet, avant la parution de l'ouvrage, Toomer a publié plusieurs extraits de Cane dans des revues littéraires, certaines clairement associées au New Negro movement, d'autres cherchant à publier avant tout une littérature moderniste innovante. Parmi ces dernières, John K. Young s'intéresse surtout à The Double Dealer, revue publiée à la Nouvelle-Orléans entre 1921 et 1926 et dont l'ambition était d'établir un dialogue entre les cultures blanche et noire et de promouvoir une littérature moderniste d'envergure nationale. Après avoir pris soin de fournir quelques éléments théoriques permettant de nourrir la réflexion sur l'objet matériel, littéraire et culturel qu'est le magazine littéraire et après avoir rappelé l'importance du réseau de lecteurs nécessairement lié à ce type de revues, John K. Young explique que le mouvement moderniste, quoiqu'associé aux grandes villes américaines de la côte Est et à quelques capitales européennes, s'est aussi développé dans des zones périphériques, comme le 
prouve la parution à la Nouvelle-Orléans de la revue The Double Dealer, dont un bref historique est proposé. Ces jalons théoriques permettent de mieux comprendre la manière originale dont John $\mathrm{K}$. Young propose de lire rétrospectivement les fragments de Cane parus dans The Double-Dealer, à la fois comme des extraits d'une œuvre complète à paraitre et comme des œuvres dont l'existence individuelle doit être reconnue, ne serait-ce que parce qu'elles (ap)paraissent dépourvues de toute référence à la question raciale et au Sud et de toute suggestion quant à la couleur de peau de leur auteur. De ce fait, l'hypothèse de départ de John $\mathrm{K}$. Young selon laquelle Cane appartient à la fois au New Negro movement mais aussi à un mouvement moderniste plus large peut être confirmée.

Dans sa contribution, Laurence Cossu-Beaumont, qui situe sa recherche dans la lignée de l'ouvrage fondateur de John K. Young sur la relation entre auteurs noirs et éditeurs blancs $^{2}$, s'intéresse à la promotion et à la diffusion de la littérature noire par l'intermédiaire des très populaires clubs de lecture américains. Son étude porte sur le cas de Richard Wright, dont les œuvres Native Son (1940) et Black Boy (1945) furent inscrites au catalogue du très conservateur Book-of-the-Month Club dans les années 1940, et sur le cas de Toni Morrison dont les romans Song of Solomon (1977) et Beloved (1991) furent sélectionnés par Oprah's Book Club, du nom de la célèbre présentatrice de télévision Oprah Winfrey. L'intérêt de l'étude est de montrer quelles stratégies ces clubs de lecture ont pu déployer pour faire de ces œuvres, a priori destinées à un public restreint, des best-sellers. À propos de Native Son, roman dans lequel la question raciale et la critique de la politique ségrégationniste des États-Unis occupent une place centrale, Laurence Cossu-Beaumont explique comment le Book-of-the-Month Club est parvenu à dénaturer le texte de Wright en exigeant de l'auteur qu'il procède à des coupes dans certains passages. De même, en faisant la promotion du livre de manière à présenter le héros du roman, Bigger, non comme un héros afro-américain mais simplement américain, le club entendait toucher un public beaucoup plus large que celui auquel le livre de Wright semblait initialement destiné. Wright résista cependant à la remise en cause de son autorité en signant une introduction à son roman intitulée «How Bigger Was Born» qui fut ajoutée aux rééditions distribuées par la maison d'édition Harper, limitant ainsi le processus de «standardisation » subi par son œuvre et par son héros. Dans le cas de Toni Morrison, Oprah Winfrey fit intervenir l'auteur dans son émission télévisée afin d'asseoir son autorité et, par là même, sa popularité, mais la présentatrice réussit néanmoins à faire prévaloir son point de vue personnel sur l'œuvre. De fait, Winfrey parvint à donner envie à des téléspectateurs, a priori peu familiers de la "grande" littérature, d'acheter les livres de Morrison, en utilisant comme argument de vente, selon la formule empruntée au talk show, l'idée qu'ils devaient permettre au lecteur de trouver un écho personnel à l'histoire racontée. L'article s'achève en rappelant les liens importants entre la sélection par les clubs de lecture et l'augmentation sensible des ventes de livres, lesquelles permettent à leurs auteurs d'acquérir une liberté de ton autorisée par le succès.

7 La troisième contribution de cette partie, que l'on doit à Max Cavitch, s'interroge sur les multiples implications de la relation entre identité et imprimé, en s'intéressant plus particulièrement au recueil de poésie, initialement publié en 1773 à Londres, de la première poétesse noire américaine, Phillis Wheatley. Max Cavitch pose d'abord quelques jalons historiques illustrant le rapport complexe entre l'auteur esclave, luimême marchandise dépossédée de son propre corps, et son œuvre consignée dans un objet-livre a priori hors d'atteinte. Puis Max Cavitch concentre son analyse sur les 
rééditions de l'œuvre de Wheatley, notamment celles réalisées par des abolitionnistes dans les années 1830 pour servir leur cause. Certaines de ces éditions combinent l'œuvre de Wheatley à celle d'un autre auteur, à l'exemple du poète noir George Moses Horton dans l'édition de 1838 publiée par Isaac Knapp. Cavitch montre que l'objectif visé par les abolitionnistes était de mettre en avant les qualités humaines et intellectuelles de ces poètes noirs, dans le but avoué de promouvoir l'intégration des esclaves, une fois affranchis, à la société américaine. Mais il voit également dans la conjonction de l'œuvre de Wheatley, produite à la fin du xvIII e siècle, et celle de Horton écrite en 1837, la volonté de faire de Wheatley l'instigatrice d'une tradition littéraire noire, qui assoit par là même son autorité en tant qu'auteur et en tant qu'être humain. La dernière partie de l'article se présente comme un décodage critique de la couverture de l'ouvrage de Henry Louis Gates Jr. intitulé The Trials of Phyllis Wheatley et publié en 2003, qui permet à Max Cavitch de conclure sur la réappropriation de l'œuvre de Wheatley et sur sa canonisation à l'époque contemporaine.

L'ouvrage se referme sur un épilogue en forme de témoignage écrit par Samuel Blumenfeld, aujourd'hui chroniqueur au journal Le Monde, qui relate la manière dont il a contribué dans les années 1990, notamment avec Marc Gerald, à faire sortir de l'anonymat et de l'invisibilité des œuvres produites dans les années 1970 par des auteurs noirs américains. Samuel Blumenfeld explique en particulier comment ces œuvres, appartenant au genre de la fiction policière pour l'essentiel, et donc considérées comme une "sous-littérature ", ont nécessité de longues recherches quasi archéologiques pour être retrouvées et rééditées au sein de deux séries, "Soul Fiction" publiée par les Éditions de l'Olivier en France et «Old School Books », publiée par W. W. Norton aux États-Unis. L'occasion est de nouveau fournie, s'il en était besoin, de rappeler l'importance du dialogue transatlantique dans une discipline telle que l'histoire du livre, dont il nous est également de nouveau donné de voir les incommensurables possibilités en tant que domaine de recherche.

\section{NOTES}

1. Sur l'histoire du livre et les études afro-américaines, voir Laurence Cossu-Beaumont et Claire Parfait, « Book History and African American Studies », Transatlantica [En ligne], 1 | 2009, mis en ligne le 23 juin 2009, consulté le 24 février 2015. URL : http://transatlantica.revues.org/4280

2. John K. Young, Black Writers, White Publishers: Marketplace Politics in Twentieth-Century African American Literature, Jackson, University Press of Mississippi, 2006. 


\section{AUTHORS}

\section{SANDRINE FERRÉ-RODE}

Université de Versailles - Saint-Quentin-en-Yvelines 